\title{
Existence theory and approximate solution to prey-predator coupled system involving nonsingular kernel type derivative
}

\author{
Manar A. Alqudah', Thabet Abdeljawad ${ }^{2,3,4^{*}}$ (D), Eiman ${ }^{5}$, Kamal Shah $^{5}$, Fahd Jarad ${ }^{6}$ and \\ Qasem Al-Mdallal ${ }^{7 *}$ (ID
}

${ }^{*}$ Correspondence:

tabdeljawad@psu.edu.sa:

q.almdallal@uaeu.ac.ae

2 Department of Mathematics and

General Sciences, Prince Sultan

University, Riyadh, Saudi Arabia

${ }^{7}$ Department of Mathematical

Sciences, UAE University, P.O. Box

15551, Al Ain, United Arab Emirates

Full list of author information is

available at the end of the article

\section{Springer}

\begin{abstract}
This manuscript considers a nonlinear coupled system under nonsingular kernel type derivative. The considered problem is investigated from two aspects including existence theory and approximate analytical solution. For the concerned qualitative theory, some fixed point results are used. While for approximate solution, the Laplace transform coupled with Adomian method is applied. Finally, by a pertinent example of prey-predator system, we support our results. Some graphical presentations are given using Matlab.
\end{abstract}

MSC: Primary 26A33; secondary 34A07; $93 \mathrm{~A} 30$

Keywords: Prey-predator system; Existence theory; Laplace transform coupled with Adomian method

\section{Introduction}

In the last few decades significant interest has been shown in fractional calculus by researchers of different disciplines of science and engineering. The concerned area has many applications in modeling various real world problems, since fractional derivative is usually a definite integral including classical derivative as a special case. Also it geometrically produces the whole spectrum or accumulation of a function. The aforesaid derivative has been defined by a number of ways. In other words, various mathematicians have given different definitions of fractional order derivative [1]. The most notable definitions were given by Riemann-Liouville and Caputo, those definitions have been increasingly used in applications in the last decades, for details, see [2-4]. In fact fractional order differential equations (FODEs) have many applications in mathematical modeling of chemical, physical, and biological phenomena. The mentioned differential operator has the ability to describe many features of hereditary and memory materials more explicitly than that of classical order. Therefore significantly FODEs have been used in the last few decades in modeling various processes and phenomena (see for applications [5-16]).

Mathematical models are strong material to investigate many phenomena. For instance, occasionally in the previous time in many localities, unforeseen large potion in fish and

(c) The Author(s) 2020. This article is licensed under a Creative Commons Attribution 4.0 International License, which permits use sharing, adaptation, distribution and reproduction in any medium or format, as long as you give appropriate credit to the original author(s) and the source, provide a link to the Creative Commons licence, and indicate if changes were made. The images or other third party material in this article are included in the article's Creative Commons licence, unless indicated otherwise in a credit line to the material. If material is not included in the article's Creative Commons licence and your intended use is not permitted by statutory regulation or exceeds the permitted use, you will need to obtain permission directly from the copyright holder. To view a copy of this licence, visit http://creativecommons.org/licenses/by/4.0/. 
animal takeover had been reported. This phenomenon gave birth to the well-known preypredator relationship. Therefore, in 1920, Lotka and Volterra introduced their famous equations known as prey-predator model. Here we state that the concerned model given in (1) deals with the relationship of prey and predator in an ecological system as follows:

$$
\left\{\begin{array}{l}
\dot{u}(t)=a_{1}(t) u(t)-b_{1}(t) v(t) u(t)=\varphi_{1}(t, u(t), v(t)), \\
\dot{v}(t)=a_{2}(t) u(t) v(t)-b_{2}(t) v(t)=\varphi_{1}(t, u(t), v(t)), \\
u(0)=\alpha, \quad v(0)=\beta,
\end{array}\right.
$$

where $\alpha, \beta \geq 0$. Further the nonlinear functions $\varphi_{i}(i=1,2): \mathcal{J} \times \mathcal{R}^{2} \rightarrow \mathcal{R}$ are continuous. Here we remark that $u(t), v(t)$ represent the prey and the predator populations at time $t$ respectively. Further $a_{1}$ is the growth rate of species $u$, while $b_{1}$ denotes the impact of predation on $\dot{u} / u$. Also $b_{2}$ is the death rate of $v$ and $a_{2}$ is the growth rate (or immigration) of the predator population in response to the size of the prey population. The coefficients are linear continuous and bounded functions. So far the concerned model has been studied for various purposes and from various directions; for details, we refer to [17-19]. Also, model (1) has been investigated by using the homotopy perturbation method for ordinary Caputo derivative in [20].

Here, we remark that the definitions of fractional derivative were further extended from singular kernel to nonsingular kernel by Caputo and Fabrizio [21] in 2016. This definition has got much attention in the last few years. Some valuable results were investigated in $[22,23]$. Further the aforesaid definition was generalized by replacing exponential kernel with Mittag-Leffler kernel. In this regard lots of research articles have been published, we refer to [24-26].

Motivated by the aforesaid work, in this work we undertake model (1) under CFFD as follows:

$$
\left\{\begin{array}{l}
{ }^{C F} D_{t}^{\omega} u(t)=a_{1}(t) u(t)-b_{1}(t) u(t) v(t), \\
{ }^{C F} D_{t}^{\omega} v(t)=a_{2}(t) u(t) v(t)-b_{2}(t) v(t), \\
u(0)=\alpha, \quad v(0)=\beta
\end{array}\right.
$$

where $\alpha, \beta \geq 0$ and $\omega \in(0,1]$.

In the last two decades, to handle FODEs for their exact or numerical solutions, various techniques, methods, and theories were established. Because finding exact analytical solutions for every differential equation of fractional order is quite a difficult job, the wellknown techniques including homotopy perturbation method, Adomian decomposition method, and many other numerical methods were utilized for the required results (for details, see [27]). As we know, the mentioned techniques were increasingly adopted for ordinary FODEs, but there are very few articles which study decompositions techniques coupled with integral transform for FODEs under CFFD, see [28-32].

Therefore, here, we construct existence theory of solution to the following semianalytical results to the coupled system with $t \in \mathcal{J}=[0, \tau]$ for the given prey-predator system in (2).

First we establish some qualitative results as the existence and uniqueness of the solution corresponding to the model we have considered. Fixed point theory is used to get these 
results about solution due to Krasnoselskii and Banach. Also some approximate analytical results are established via the Laplace transform and Adomian decomposition tools. The concerned approximate results are illustrated by graphs via Matlab.

We arrange our paper as follows: In Sect. 1, we give introduction to the problem. In Sect. 2, we recall some preliminaries. In Sect. 3, we establish the existence results of the main work. In Sect. 4, we present general procedure for approximate solution. Finally, in Sect. 5, we give examples and brief conclusion.

\section{Preliminaries}

Definition $1([22,23])$ Let $\varphi \in \mathcal{H}^{1}(0, \tau), \tau>0, \omega \in(0,1)$, then the CFFD is defined as follows:

$$
{ }^{C F} \mathcal{D}_{t}^{\omega}(\varphi(t))=\frac{\mathscr{M}(\omega)}{1-\omega} \int_{0}^{t} \varphi^{\prime}(t) \exp \left[-\omega \frac{t-\xi}{1-\omega}\right] d \xi
$$

$\mathscr{M}(\omega)=\frac{2 \omega}{2-\omega}$ is known as a normalization function and statistics $\mathscr{M}(1)=\mathscr{M}(0)=1$. Moreover, if $\varphi$ does not fall in $\mathcal{H}^{1}(0, \tau)$, then the derivatives are given by

$$
{ }^{C F} \mathcal{D}_{t}^{\omega}(\varphi(t))=\frac{\mathscr{M}(\omega)}{1-\omega} \int_{0}^{t}(\varphi(t)-\varphi(\xi)) \exp \left[-\omega \frac{z-\xi}{1-\omega}\right] d \xi
$$

Definition 2 ([22]) For $\varphi \in \mathcal{H}^{1}(0, \tau), \tau>0$, the integral in Caputo-Fabrizio form is given as follows:

$$
{ }^{C F} \mathcal{I}_{t}^{\omega}[\varphi(t)]=\frac{(1-\omega)}{\mathscr{M}(\omega)} \varphi(t)+\frac{\omega}{\mathscr{M}(\omega)} \int_{0}^{t} \varphi(\xi) d \xi, \quad \omega \in(0,1] .
$$

Definition 3 ([22]) The Laplace transform of ${ }^{C F} \mathcal{D}_{t}^{\omega} u(t)$ with $\mathscr{M}(\omega)=1$ is given as follows:

$$
\mathscr{L}\left[{ }^{C F} \mathcal{D}_{t}^{\omega} u(t)\right]=\frac{s \mathscr{L}[u(t)]-u(0)}{s+\omega(1-s)}, \quad s \geq 0, \omega \in(0,1]
$$

Note Corresponding to existence theory, let $\mathcal{J}=[0, \tau]$ and $0 \leq t \leq \tau<\infty$, we define the space as $\mathbf{Z}=C\left([0, \tau] \times \mathcal{R}^{2}, \mathcal{R}\right)$ equipped with the norm $\|(u, v)\|=\sup _{t \in \mathcal{J}}[|u(t)|+|v(t)|]$.

Theorem 1 ([33]) Let $\mathbb{B}$ be a convex subset of $\mathbf{Z}$, and we have two operators $\mathbb{F}, \mathbb{G}$ with

1. $\mathbb{F} w+\mathbb{G} w \in \mathbb{B}$ for every $w \in \mathbb{B}$;

2. $\mathbb{F}$ is a contraction;

3. $\mathbb{G}$ is continuous and compact.

Then the operator equation $\mathbb{F} w+\mathbb{G} w=w$ has at least one solution.

\section{Existence and uniqueness results of fractional order predator-prey equations}

In this part some results about existence and uniqueness are given about the solution of the proposed model (2). Upon using integral operator ${ }^{C F} \mathcal{I}_{t}^{\omega}$ on both sides of (2) and putting the initial conditions, one has

$$
\left\{\begin{array}{l}
u(t)=\alpha+\frac{(1-\omega)}{\mathscr{M}(\omega)} \varphi_{1}(t, u, v)+\frac{\omega}{\mathscr{M}(\omega) \Gamma(\omega)} \int_{0}^{t}(t-\xi)^{\omega-1} \varphi_{1}(\xi, u(\xi), v(\xi)) d \xi \\
\beta(t)=\beta+\frac{(1-\omega)}{\mathscr{M}(\omega)} \varphi_{2}(t, u, v)+\frac{\omega}{\mathscr{M}(\omega) \Gamma(\omega)} \int_{0}^{t}(t-\xi)^{\omega-1} \varphi_{2}(\xi, u(\xi), v(\xi)) d \xi
\end{array}\right.
$$


which further may be written as

$$
\mathbf{W}(t)=\mathbf{W}_{0}+\Psi(t, \mathbf{W}(t)) \frac{(1-\omega)}{\mathscr{M}(\omega)}+\frac{\omega}{\mathscr{M}(\omega)} \int_{0}^{t} \Psi(\xi, \mathbf{W}(\xi)) d \xi,
$$

where

$$
\mathbf{W}(t)=\left\{\begin{array}{l}
u(t), \\
v(t),
\end{array} \quad \mathbf{W}_{0}=\left\{\begin{array}{l}
\alpha, \\
\beta,
\end{array} \quad \Psi(t, \mathbf{W}(t))=\left\{\begin{array}{l}
\varphi_{1}(t, u, v), \\
\varphi_{2}(t, u, v)
\end{array}\right.\right.\right.
$$

Now, to derive our results, we define the following assumptions:

(A1) There exist constants $\mathbf{L}_{\Psi}>0$ such that, for each $\mathbf{W}, \overline{\mathbf{W}} \in \mathbf{Z}$,

$$
|\Psi(t, \mathbf{W}(t))-\Psi(t, \overline{\mathbf{W}}(t))| \leq \mathbf{L}_{\Psi}[|\mathbf{W}-\overline{\mathbf{W}}|]
$$

(A2) There exist constants $C_{\Psi}, C_{\Psi}>0$ and $\mathbf{M}_{\Psi}>0$ such that

$$
|\Psi(t, \mathbf{W}(t))| \leq C_{\Psi}|\mathbf{W}|+M_{\Psi}
$$

Using (4) and (5), the operators are defined as follows:

$$
\begin{aligned}
& \mathbb{F}(\mathbf{W})=\mathbf{W}_{0}(t)+\Psi(t, \mathbf{W}(t)) \frac{(1-\omega)}{\mathscr{M}(\omega)}, \\
& \mathbb{G}(\mathbf{W})=\frac{\omega}{\mathscr{M}(\omega)} \int_{0}^{t} \Psi(\xi, \mathbf{W}(\xi)) d \xi
\end{aligned}
$$

Theorem 2 With the help of (A1) and (A2), the integral system (4) has at least one solution provided that $\frac{\mathrm{L}_{\Psi}}{\mathscr{M}(\omega)}<1$.

Proof Let $\mathbb{B}=\{\mathbf{W} \in \mathbf{Z}:\|\mathbf{W}\| \leq \rho, \rho>0\}$ be a closed and convex subset of $\mathbf{Z}$, we need to prove that $\mathbb{F}: \mathbb{B} \rightarrow \mathbb{B}$ is a contraction. Let $\mathbf{W}-\overline{\mathbf{W}} \in \mathbb{B}$, we have

$$
\begin{aligned}
\|\mathbb{F} \mathbf{W}-\mathbb{F} \overline{\mathbf{W}}\| & =\sup _{t \in \mathcal{J}} \mid\left(\Psi(t, \mathbf{W}(t))-(\Psi(t, \overline{\mathbf{W}}(t))) \frac{(1-\omega)}{\mathscr{M}(\omega)} \mid\right. \\
& \leq \frac{(1-\omega)}{\mathscr{M}(\omega)} \mathbf{L}_{\Psi} \sup _{t \in \mathcal{J}}|\mathbf{W}(t)-\overline{\mathbf{W}}(t)| \\
& \leq \frac{\mathbf{L}_{\Psi}}{\mathscr{M}(\omega)}\|\mathbf{W}-\overline{\mathbf{W}}\| .
\end{aligned}
$$

Hence $\mathbb{F}$ is a contraction.

For $\mathbb{G}$ to be compact and continuous, let any $\mathbf{W} \in \mathbb{B}$, we have

$$
\begin{aligned}
\|\mathbb{G}(\mathbf{W})\| & =\sup _{t \in \mathcal{J}}\left|\frac{\omega}{\mathscr{M}(\omega)} \int_{0}^{t} \Psi(\xi, \mathbf{W}(\xi)) d \xi\right| \\
& \leq \frac{\tau}{\mathscr{M}(\omega)}\left[C_{\Psi} \rho+\mathbf{M}_{\Psi}\right]:=\Delta .
\end{aligned}
$$

From (7) we conclude that $\mathbb{G}$ is bounded. Also $\Psi$ is continuous, so is $\mathbb{G}$. Along the same lines, we can prove that $\mathbb{G}$ is equicontinuous by taking $t_{1}<t_{2} \in \mathcal{J}$. By using Arzelá-Ascoli 
theorem, the operator $\mathbb{G}$ is completely continuous operator and also uniformly bounded as proved already. Hence $\mathbb{G}$ is relatively compact. By Krasnoselskii's fixed point theorem, problem (4) has at least one solution. Consequently, the considered system 2 has at least one solution.

Theorem 3 Under assumption (A1), integral system (4) has unique solution if $\frac{(1+\tau) \mathbf{L}}{\mathscr{M}(\omega)}<1$. Consequently, the system under consideration has unique solution.

Proof Let us define $\mathbf{T}: \mathbf{Z} \rightarrow \mathbf{Z}$ by

$$
\mathbf{T}(\mathbf{W})=\mathbf{W}_{0}+\Psi(t, \mathbf{W}(t)) \frac{(1-\omega)}{\mathscr{M}(\omega)}+\frac{\omega}{\mathscr{M}(\omega)} \int_{0}^{t} \Psi(\xi, \mathbf{W}(\xi)) d \xi
$$

Let $\mathbf{W}, \overline{\mathbf{W}} \in \mathbf{Z}$, we have

$$
\begin{aligned}
\|\mathbf{T}(\mathbf{W})-\mathbf{T}(\overline{\mathbf{W}})\| \leq & \sup _{t \in \mathcal{J}} \frac{(1-\omega)}{\mathscr{M}(\omega)}|\Psi(t, \mathbf{W}(t))-\Psi(t, \overline{\mathbf{W}}(t))| \\
& +\frac{\omega}{\mathscr{M}(\omega)} \sup _{t \in \mathcal{J}} \int_{0}^{t}|\Psi(\xi, \mathbf{W}(\xi))-\Psi(\xi, \overline{\mathbf{W}}(\xi))| d \xi \\
\leq & \frac{(1+\tau) \mathbf{L}}{\mathscr{M}(\omega)}\|\mathbf{W}-\overline{\mathbf{W}}\| .
\end{aligned}
$$

Hence $\mathbf{T}$ is a contraction and the concerned problem (4) has unique solution, and so the considered model (2) has unique solution.

\section{Approximate solutions to predator-prey equations (2)}

To compute the required approximate solution, for easiness, take $\mathscr{M}(\omega)=1$. Using the Laplace transform on both sides of system (2), we have

$$
\left\{\begin{array}{l}
\mathscr{L}[u(t)]=\frac{u(0)}{s}+\frac{s+\omega(1-s)}{s} \mathscr{L}\left[a_{1} u(t)-b_{1} u(t) v(t)\right], \\
\mathscr{L}[v(t)]=\frac{v(0)}{s}+\frac{s+\omega(1-s)}{s} \mathscr{L}\left[a_{2} u(t) v(t)-b_{2} v(t)\right] .
\end{array}\right.
$$

Now assume the solution in the series form as follows:

$$
u(t)=\sum_{q=0}^{\infty} u_{q}(t), \quad v(t)=\sum_{q=0}^{\infty} v_{q}(t)
$$

Further expressing the nonlinear terms $u(t) v(t)$ by using the decomposition method

$$
u(t) v(t)=\sum_{q=0}^{\infty} A_{q}(u, v)
$$

where the "Adomian polynomial" $A_{q}(u, v)$ can be defined as

$$
A_{q}(u, v)=\left.\frac{1}{q !} \frac{d^{q}}{d \lambda q}\left[\sum_{j=0}^{p} \lambda^{j} u_{j}(t) \sum_{j=0}^{p} \lambda^{j} v_{j}(t)\right]\right|_{\lambda=0} .
$$


Hence in view of (10) and (11), system (9) becomes

$$
\left\{\begin{array}{l}
\mathscr{L}\left[\sum_{q=0}^{\infty} u_{q}(t)\right]=\frac{u(0)}{s}+\frac{s+\omega(1-s)}{s} \mathscr{L}\left[a_{1}(t) \sum_{q=0}^{\infty} u_{q}(t)-b_{1}(t) \sum_{q=0}^{\infty} A_{q}(u, v)\right] \\
\mathscr{L}\left[\sum_{q=0}^{\infty} v_{q}(t)\right]=\frac{v(0)}{s}+\frac{s+\eta(1-s)}{s} \mathscr{L}\left[a_{2}(t) \sum_{q=0}^{\infty} A_{q}(u, v)-b_{2}(t) \sum_{q=0}^{\infty} v_{q}\right] .
\end{array}\right.
$$

From (12), we equate terms as follows:

$$
\left\{\begin{array}{l}
\mathscr{L}\left[u_{0}(t)\right]=\frac{u_{0}}{s}, \quad \mathscr{L}\left[v_{0}(t)\right]=\frac{v_{0}}{s}, \\
\mathscr{L}\left[u_{1}(t)\right]=\frac{s+\omega(1-s)}{s} \mathscr{L}\left[a_{1}(t) u_{0}-b_{1}(t) A_{0}(u, v)\right], \\
\mathscr{L}\left[v_{1}(t)\right]=\frac{s+\omega(1-s)}{s} \mathscr{L}\left[a_{2}(t) A_{0}(u, v)-b_{2}(t) v_{0}\right], \\
\mathscr{L}\left[u_{2}(t)\right]=\frac{s+\omega(1-s)}{s} \mathscr{L}\left[a_{1}(t) u_{1}-b_{1}(t) A_{1}(u, v)\right], \\
\mathscr{L}\left[v_{2}(t)\right]=\frac{s+\omega(1-s)}{s} \mathscr{L}\left[a_{2}(t) A_{1}(u, v)-b_{2}(t) v_{1}\right], \\
\mathscr{L}\left[u_{3}(t)\right]=\frac{s+\omega(1-s)}{s} \mathscr{L}\left[a_{1}(t) u_{2}-b_{1}(t) A_{2}(u, v)\right], \\
\mathscr{L}\left[v_{3}(t)\right]=\frac{s+\omega(1-s)}{s} \mathscr{L}\left[a_{2}(t) A_{2}(u, v)-b_{2}(t) v_{2}\right], \\
\vdots \\
\mathscr{L}\left[u_{q+1}(t)\right]=\frac{s+\omega(1-s)}{s} \mathscr{L}\left[a_{1}(t) u_{q}-b_{1}(t) A_{q}(u, v)\right], \\
\mathscr{L}\left[v_{q+1}(t)\right]=\frac{s+\omega(1-s)}{s} \mathscr{L}\left[a_{2}(t) A_{q}(u, v)-b_{2}(t) v_{q}\right], \quad q \geq 0 .
\end{array}\right.
$$

Case I:

In the first case we take coefficients as constant functions $a_{1}(t)=a_{1}, b_{1}(t)=b_{1}, c_{1}(t)=c_{1}$, $d_{1}(t)=d_{1}$ in (13). After performing simplification, we get

$$
\left\{\begin{aligned}
u_{0}(t)= & \alpha, \quad v_{0}(t)=\beta, \\
u_{1}(t)= & {\left[a_{1} \alpha-b_{1} \alpha \beta\right](1+\omega(t-1)), } \\
v_{1}(t)= & (1+\omega(t-1))\left[a_{2} \alpha \beta-b_{2} \beta\right], \\
u_{2}(t)= & {\left[\left(a_{1}-b_{1} \beta\right)\left(a_{1} \alpha-b_{1} \alpha \beta\right)-b_{1} \alpha\left(a_{2} \alpha \beta-b_{2} \beta\right)\right]\left(1+\omega^{2}(t-1)\right), } \\
v_{2}(t)= & {\left[\left(\alpha a_{2}-b_{2}\right)\left(a_{2} \alpha \beta-b_{2} \beta\right)+a_{2} \beta\left(a_{2} \alpha \beta-b_{2} \beta\right)\right]\left(1+\omega^{2}(t-1)\right), } \\
u_{3}(t)= & {\left[\left(a_{1}-b_{1} \beta\right)\left(\left(a_{1}-b_{1} \beta\right)\left(a_{1} \alpha-b_{1} \alpha \beta\right)-b_{1} \alpha\left(a_{2} \alpha \beta-b_{2} \beta\right)\right)\right.} \\
& \left.-b_{1} \alpha\left(\left(\alpha a_{2}-b_{2}\right)\right)-b_{1}\left(a_{1} \alpha-b_{1} \alpha \beta\right)\left(a_{2} \alpha \beta-b_{2} \beta\right)\right]\left(1+\omega^{3}(t-1)\right), \\
v_{3}(t)= & {\left[\left(a_{2} \alpha-b_{2}\right)\left(\left(\alpha a_{2}-b_{2}\right)\left(a_{2} \alpha \beta-b_{2} \beta\right)+a_{2} \beta\left(a_{2} \alpha \beta-b_{2} \beta\right)\right)\right.} \\
& \left.-b_{1} \alpha\left(a_{2} \alpha \beta-b_{2} \beta\right)\right)+a_{2}\left(a_{1} \alpha-b_{1} \alpha \beta\right)\left(a_{2} \alpha \beta-b_{2} \beta\right) \\
& +a_{2} \beta\left(\left(a_{1}-b_{1} \beta\right)\left(a_{1} \alpha-b_{1} \alpha \beta\right)\right]\left(1+\omega^{3}(t-1)\right)
\end{aligned}\right.
$$

and so on. In this way the other terms are computed.

Case II:

Here, we take some coefficients as linear functions $a_{1}(t)=t, b_{2}(t)=t$ and $a_{2}$ and $b_{1}$ are constants. We obtain the resultant solution as follows:

$$
\left\{\begin{array}{l}
u_{0}(t)=\alpha, \quad v_{0}(t)=\beta \\
u_{1}(t)=\alpha\left(t+\omega\left(\frac{t^{2}}{2 !}-t\right)\right)-b_{1} \alpha \beta(1+\omega(t-1)) \\
v_{1}(t)=(1+\omega(t-1)) a_{2} \alpha \beta-\beta\left(t+\omega\left(\frac{t^{2}}{2 !}-t\right)\right)
\end{array}\right.
$$


and so on. The remaining terms may similarly be computed. The required solutions in both cases will be written as

$$
\left\{\begin{array}{l}
u(t)=u_{0}+u_{1}(t)+u_{2}(t)+u_{3}(t)+\cdots, \\
v(t)=v_{0}+v_{1}(t)+v_{2}(t)+v_{3}(t)+\cdots
\end{array}\right.
$$

\section{Results and discussion}

Here, by using Matlab, we present solutions (16) up to initial ten terms by graphs using the numerical values for parameters as given in Table 1 . The solutions are displayed against various fractional orders in Figs. 1 and 2, respectively.

Further on using Matlab, we present solutions (16) up to initial ten terms by graphs using the numerical values for parameters as given in Table 2 in Case II. The solutions are displayed against various fractional orders in Figs. 3 and 4, respectively.

From Figs. 1, 3 we see that the population $u$ is deceasing at different rate due to fractional order. The smaller the order, the faster the decay process, and hence stability occurs at smaller order first and then at greater one, as compared. In the same fashion the population $v$ grows in Figs. 2 and 4, respectively, at different rate due to fractional order. The solution tends to the classical (integer) order solution when $\omega \rightarrow 1$. The solution obtained here for CFFD is close to the solution obtained by using the homotopy method in [20] by using Caputo ordinary derivatives. We have presented the solutions for both cases, i.e., Case I and Case II, in the aforesaid figures. From the figures, we conclude that CFFD can also be used as a powerful tool to investigate such systems.

Table 1 Values of parameters taken for Case I

\begin{tabular}{lll}
\hline Parameters & Description of parameters & Numerical value \\
\hline$a_{1}$ & The growth rate of prey & 0.009978 \\
$b_{1}$ & The rate at which predators destroy prey & 0.00342 \\
$a_{2}$ & The death rate of predators & 0.00342 \\
$b_{2}$ & The growth rate of predators & 0.000765 \\
$\alpha$ & The initial population of prey & 18 \\
$\beta$ & The initial population of predator & 14 \\
\hline
\end{tabular}

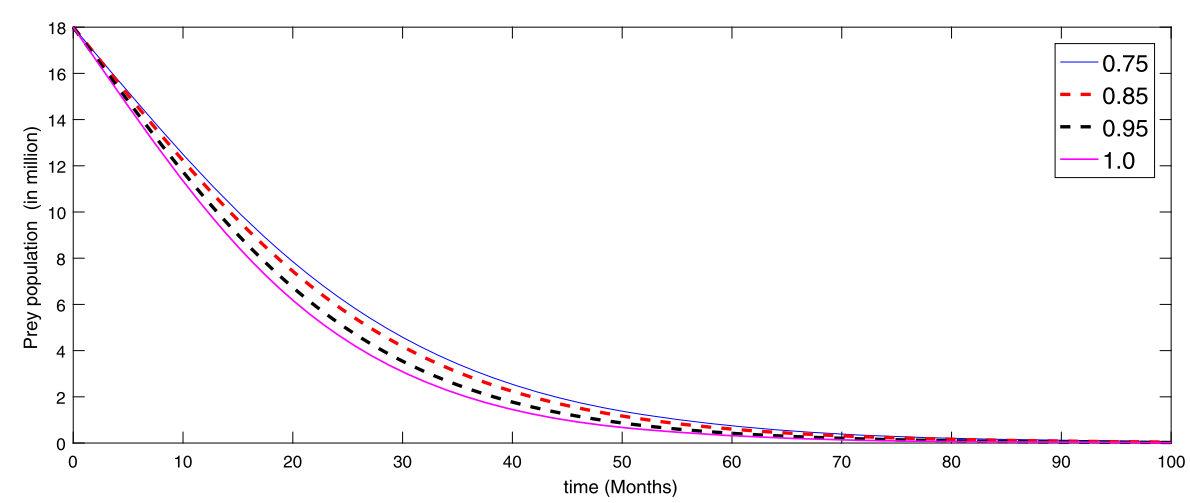

Figure 1 Graphical representation of the approximate solution $u$ for different fractional order taking initial ten terms of the series in Case I 


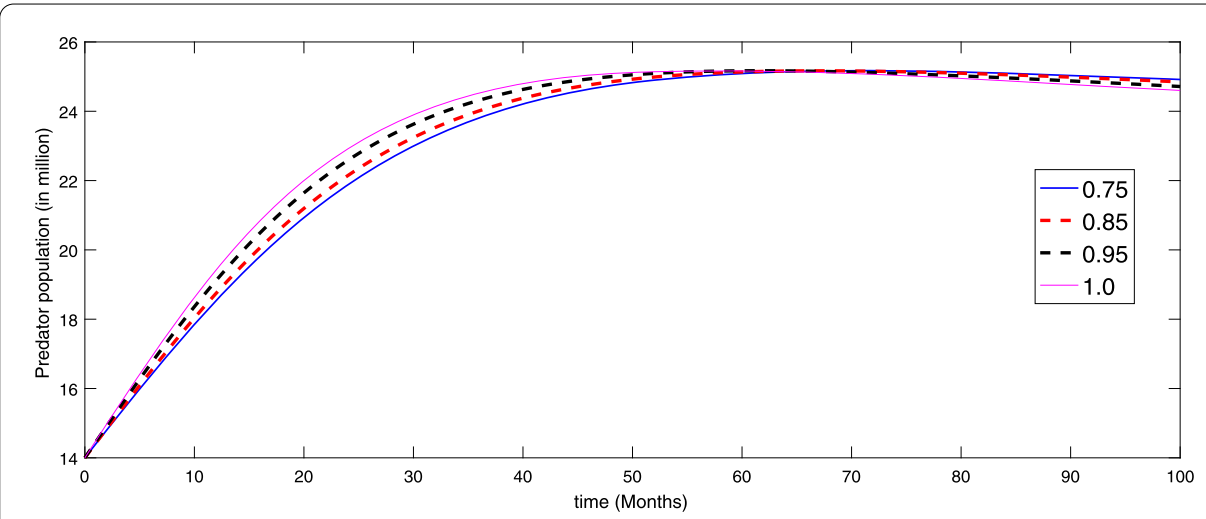

Figure 2 Graphical representation of the approximate solution $v$ for different fractional order taking initial ten terms of the series in Case I

Table 2 Values of parameters taken for Case II

\begin{tabular}{lll}
\hline Parameters & Description of parameters & Numerical value \\
\hline$a_{1}(t)$ & The growth rate of prey & $t$ \\
$b_{1}$ & The rate at which predators destroy prey & 0.00342 \\
$a_{2}$ & The death rate of predators & 0.00342 \\
$b_{2}(t)$ & The growth rate of predators & $t$ \\
$\alpha$ & The initial population of prey & 18 \\
$\beta$ & The initial population of predator & 14 \\
\hline
\end{tabular}

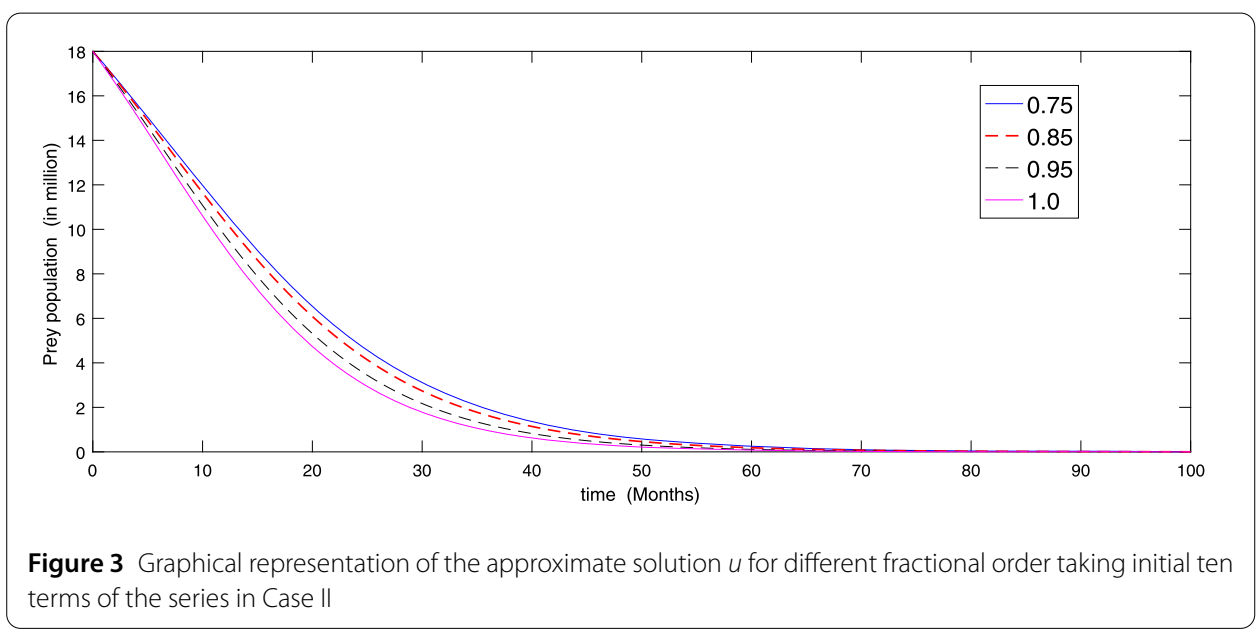

\section{Conclusion}

Since predator-prey models are debatably the building blocks of the bio- and ecosystems in which both the species depend on each other, we have taken two sets of parameter values in Tables 1 and 2. We have graphed the approximate solutions for different fractional order in Figs. 1-4, respectively. We see that the population of predators increases as shown in Figs. 2, 4, respectively. The growth rate is faster at smaller fractional order, and as the order increases, the solution behavior coincides with the solution at integer order. Consequently, the population of prey goes on deceasing as in Figs. 1 and 3. The decay rate is faster on smaller fractional order, while slower on greater order. Hence, the dynamical system addressing the relationship between prey and predator has been investigated under CFFD 


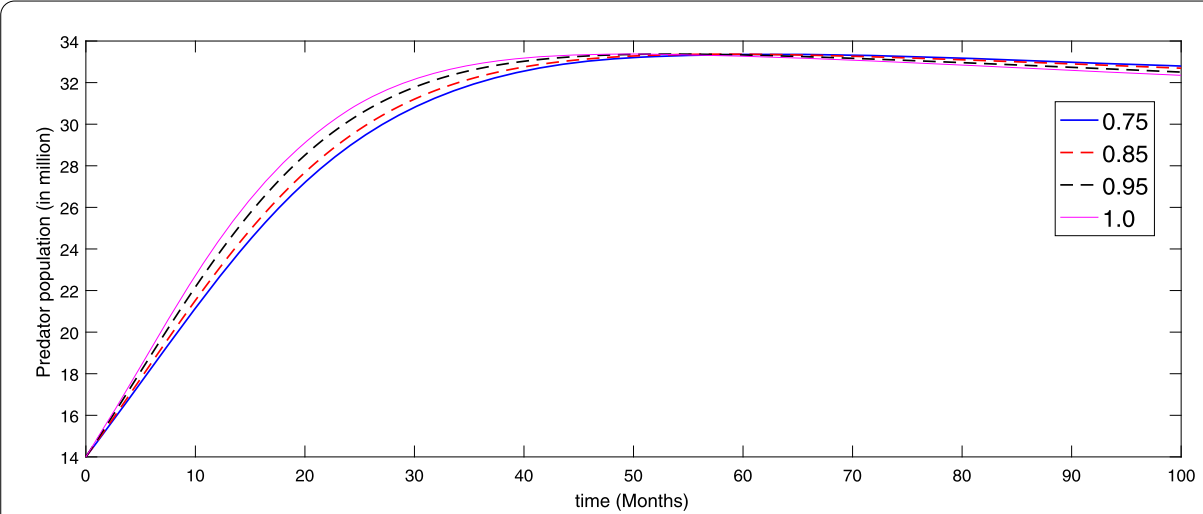

Figure 4 Graphical representation of the approximate solution $v$ for different fractional order taking initial ten terms of the series in Case II

from qualitative and analytical aspects. By using fixed point approach, the existence of the model has been verified. Also, by combining the Laplace transform with the decomposition method, some approximate analytical results have been established under two cases. In the first case for constant coefficients and in Case II the concerned results have been obtained for variable coefficients. Hence we concluded that taking few terms of the series solutions we can efficiently describe the model under investigation.

\section{Funding}

This research was funded by the Deanship of Scientific Research at Princess Nourah bint Abdulrahman University through the Fast-track Research Funding Program.

\section{Availability of data and materials}

Data sharing is not applicable to this article as no data sets were generated or analyzed during the current study.

\section{Competing interests}

The authors declare that they have no competing interests.

\section{Authors' contributions}

All authors equally contributed to this manuscript and approved the final version.

\section{Author details}

${ }^{1}$ Department of Mathematical Sciences, Faculty of Science, Princess Nourah Bint Abdulrahman University, P.O. Box 84428, Riyadh 11671, Saudi Arabia. ${ }^{2}$ Department of Mathematics and General Sciences, Prince Sultan University, Riyadh, Saudi Arabia. ${ }^{3}$ Department of Medical Research, China Medical University, Taichung 40402, Taiwan. ${ }^{4}$ Department of Computer Science and Information Engineering, Asia University, Taichung, Taiwan. ${ }^{5}$ Department of Mathematics, University of Malakand, Dir(L), Khyber Pakhtunkhwa, Pakistan. ${ }^{6}$ Department of Mathematics, Cankaya University, Ankara, Turkey. ${ }^{7}$ Department of Mathematical Sciences, UAE University, P.O. Box 15551, Al Ain, United Arab Emirates.

\section{Publisher's Note}

Springer Nature remains neutral with regard to jurisdictional claims in published maps and institutional affiliations.

Received: 19 July 2020 Accepted: 13 September 2020 Published online: 24 September 2020

\section{References}

1. Naghipour, A., Manafian, J.: Application of the Laplace Adomian decomposition method and implicit methods for solving Burgers' equations. TWMS J. Pure Appl. Math. 6(1), 68-77 (2015)

2. Thieme, H.R.: Convergence results and a Poincaré-Bendixson trichotomy for asymptotically autonomous differential equations. J. Math. Biol. 30(7), 755-763 (1992)

3. Biazar, J.: Solution of the epidemic model by Adomian decomposition method. Appl. Math. Comput. 173(2), 1101-1106 (2006)

4. Podlubny, l.: Fractional Differential Equations. Mathematics in Science and Engineering. Academic Press, New York (1999)

5. Kilbas, A.A., Srivastava, H., Trujillo, J.: Theory and Application of Fractional Differential Equations. North Holland Mathematics Studies, vol. 204. Elsevier, Amsterdam (2006) 
6. Abdon, A.: Fractal-fractional differentiation and integration: connecting fractal calculus and fractional calculus to predict complex system. Chaos Solitons Fractals 102, 396-406 (2017)

7. Yudong, Z., et al.: Tea category identification using a novel fractional Fourier entropy and Jaya algorithm. Entropy $18(3), 77(2016)$

8. Jagdev, S., et al:: A fractional epidemiological model for computer viruses pertaining to a new fractional derivative. Appl. Math. Comput. 316, 504-515 (2018)

9. Ndolane, S.: Second-grade fluid model with Caputo-Liouville generalized fractional derivative. Chaos Solitons Fractals 133, 109631 (2020)

10. Abdon, A.: Fractional discretization: the African's tortoise walk. Chaos Solitons Fractals 130, 109399 (2020)

11. El-Saka, A.A.A.: The fractional-order SIS epidemic model with variable population size. J. Egypt. Math. Soc. 22, 50-54 (2014)

12. Gomez-Aguilar, J.F., et al.: Analysis of fractal-fractional malaria transmission model. Fractals (2020). https://doi.org/10.1142/S0218348X20400411

13. Ivorra, B., et al.: Mathematical modeling of the spread of the coronavirus disease 2019 (COVID-19) considering its particular characteristics. The case of China. Commun. Nonlinear Sci. Numer. Simul. 88, 105303 (2020)

14. Yan, D., Cao, H.: The global dynamics for an age-structured tuberculosis transmission model with the exponential progression rate. Appl. Math. Model. 75, 769-786 (2019)

15. Kucharski, A.J., Russell, T.W., Diamond, C., Liu, Y., Edmunds, J., Funk, S., et al.: Early dynamics of transmission and control of COVID-19: a mathematical modelling study. Lancet Infect. Dis. (2020). https://doi.org/10.1016/S1473-3099(20)30144-4

16. Matignon, D.: Stability results for fractional differential equations with applications to control processing. In: Computational Engineering in Systems Applications, vol. 2, pp. 963-968 (1996)

17. Abrams, P.A.: The evolution of predator-prey interactions: theory and evidence. Annu. Rev. Ecol. Syst. 31, 79-105 (2000)

18. Berryman, A.: The origins and evolution of predator-prey theory. Ecology 73, 1530-1535 (1992)

19. Tahara, T., et al.: Asymptotic stability of a modified Lotka-Volterra model with small immigrations. Sci. Rep. 8(1), 7029 (2018)

20. Yiha, M.D., Koya, P.R., Tibebu, T.: Analysis of prey-predator system with prey population experiencing critical depensation growth function. Am. J. Appl. Math. 3(6), 327-334 (2015)

21. Caputo, M., Fabrizio, M.: A new definition of fractional derivative without singular kernel. Prog. Fract. Differ. Appl. 1(2), $1-13(2015)$

22. Singh, J., et al.: Analysis of an El Nino-southern oscillation model with a new fractional derivative. Chaos Solitons Fractals 99, 109-115 (2017)

23. Tarasov, V.E.: Caputo-Fabrizio operator in terms of integer derivatives: memory or distributed lag. Comput. Appl. Math. 38(3), 113 (2019)

24. Abdon, A.: Fractional discretization: the African's tortoise walk. Chaos Solitons Fractals 130, 109399 (2020)

25. Yang, X.-J., et al.: On exact traveling-wave solutions for local fractional Korteweg-de Vries equation. Chaos 26(8), $084312(2016)$

26. Abdon, A.: Blind in a commutative world: simple illustrations with functions and chaotic attractors. Chaos Solitons Fractals 114, 347-363 (2018)

27. Abdulaziz, O., Hashim, I., Momani, S.: Solving systems of fractional differential equations by homotopy-perturbation method. Phys. Lett. A 372(4), 451-459 (2008)

28. Shah, K., Alqudah, M.A., Jarad, F., Abdeljawad, T.: Semi-analytical study of Pine Wilt disease model with convex rate under Caputo-Fabrizio fractional order derivative. Chaos Solitons Fractals 135, 109754 (2020)

29. Nazir, G., Shah, K., Debbouche, A., Khan, R.A.: Study of HIV mathematical model under nonsingular kernel type derivative of fractional order. Chaos Solitons Fractals 139, 110095 (2020)

30. Haq, F., Shah, K., Rahman, G., Shahzad, M.: Numerical solution of fractional order smoking model via Laplace Adomian decomposition method. Alex. Eng. J. 57(2), 1061-1069 (2018)

31. Ali, A., Shah, K., Khan, R.A.: Numerical treatment for traveling wave solutions of fractional Whitham-Broer-Kaup equations. Alex. Eng. J. 57(3), 1991-1998 (2018)

32. Kiymaz, O.: An algorithm for solving initial value problems using Laplace Adomian decomposition method. Appl. Math. Sci. 3(30), 1453-1459 (2009)

33. Burton, T.A.: Krasnoselskii $N$-tupled fixed point theorem with applications to fractional nonlinear dynamical system Adv. Math. Phys. 2019, Article ID 6763842 (2019)

\section{Submit your manuscript to a SpringerOpen ${ }^{\circ}$ journal and benefit from:}

- Convenient online submission

- Rigorous peer review

- Open access: articles freely available online

- High visibility within the field

- Retaining the copyright to your article

Submit your next manuscript at $\gg$ springeropen.com 